\title{
BROOKHSUEN
}

NATIONAL LABORATORY

BNL-75747-2006-CP

\section{Electron Beam Ion Source Pre-Injector Diagnostics}

\author{
M. Wilinski, J. Alessi, E. Beebe; S. Bellavia, A. Pikin \\ Presented at the Beam Instrumentation Workshop 2006 \\ Batavia, IL \\ May 1-4, 2006
}

Collider-Accelerator Department

Brookhaven National Laboratory

P. O. Box 5000

Upton, NY 11973-5000

www.bnl.gov

\begin{abstract}
Notice: This manuscript has been authored by employees of Brookhaven Science Associates, LLC under Contract No. DE-AC02-98CH10886 with the U.S. Department of Energy. The publisher by accepting the manuscript for publication acknowledges that the United States Government retains a non-exclusive, paid-up, irrevocable, world-wide license to publish or reproduce the published form of this manuscript, or allow others to do so, for United States Government purposes.
\end{abstract}

This preprint is intended for publication in a journal or proceedings. Since changes may be made before publication, it may not be cited or reproduced without the author's permission. 


\section{DISCLAIMER}

This report was prepared as an account of work sponsored by an agency of the United States Government. Neither the United States Government nor any agency thereof, nor any of their employees, nor any of their contractors, subcontractors, or their employees, makes any warranty, express or implied, or assumes any legal liability or responsibility for the accuracy, completeness, or any third party's use or the results of such use of any information, apparatus, product, or process disclosed, or represents that its use would not infringe privately owned rights. Reference herein to any specific commercial product, process, or service by trade name, trademark, manufacturer, or otherwise, does not necessarily constitute or imply its endorsement, recommendation, or favoring by the United States Government or any agency thereof or its contractors or subcontractors. The views and opinions of authors expressed herein do not necessarily state or reflect those of the United States Government or any agency thereof. 


\title{
Electron Beam Ion Source Pre-Injector Diagnostics $^{*}$
}

\author{
M. Wilinski, J. Alessi, E. Beebe, S. Bellavia, A. Pikin \\ Brookhaven National Laboratory, Upton, NY 11973-5000, USA
}

\begin{abstract}
A new ion pre-injector line is currently under design at Brookhaven National Laboratory (BNL) for the Relativistic Heavy Ion Collider (RHIC) and the NASA Space Radiation Laboratory (NSRL). Collectively, this new line is referred to as the EBIS project. This pre-injector is based on an Electron Beam Ion Source (EBIS), a Radio Frequency Quadrupole (RFQ) accelerator, and a linear accelerator. The new EBIS will be able to produce a wide range of heavy ion species as well as rapidly switching between species. To aid in operation of the pre-injector line, a suite of diagnostics is currently proposed which includes faraday cups, current transformers, profile monitors, and a pepperpot emittance measurement device.
\end{abstract}

Keywords: diagnostics, instrumentation, faraday cup, profile monitor, current transformer, pepperpot, electron beam ion source

PACS: 29.17.+w, 29.25.Ni, 29.27.Fh

\section{INTRODUCTION}

The EBIS pre-injector is being constructed as a replacement to the ColliderAccelerator Department's (CAD) present pre-injector, the Tandem Van de Graaff. The Tandem was built around 1970 and is showing signs of age. Significant upgrades are required to allow the Tandem to maintain reliable operation and to be able to produce the types and intensities of ions that both RHIC and NSRL are requesting. An EBIS test stand was constructed for development and experimental results have proven that an EBIS meeting beam requirements can be successful ${ }^{1,2}$. The EBIS project received DOE critical decision 1 (CD-1) approval in September 2005 and is expected to obtain critical decisions 2 and 3 this year (2006).

TABLE 1. EBIS Parameters and Specifications.

\begin{tabular}{lc}
\hline Parameter & Specification \\
\hline Electron Beam Current & $10 \mathrm{~A}$ \\
Ion Beam Pulse Width & $10-40 \mu \mathrm{s}$ \\
Intensity in Desired Charge State & $\geq 1 \times 10^{11} \mathrm{charges} /$ pulse \\
Repetition Rate & $5 \mathrm{~Hz}$ \\
Switching Time Between Species & $1 \mathrm{~second}$ \\
Output Energy & $2 \mathrm{MeV} / \mathrm{amu}$ \\
Emittance (Full Beam, Normalized) & $\leq 1.4 \pi \mathrm{mm} \mathrm{mrad}$ \\
\hline
\end{tabular}

- Work performed under auspices of the U.S. Department of Energy 
Table 1 gives a list of some relevant specifications for the EBIS pre-injector ${ }^{3}$. The transport line will be approximately $30 \mathrm{~m}$ and will inject full intensity beam into Booster over 1-4 turns, as opposed to the 30-40 turns that it takes presently. Booster injection from the EBIS transfer line will be situated in the same place as the present Tandem injection location. The EBIS is capable of producing any ion species in contrast with the present Tandem which can only produce negative ion species. EBIS will have the potential to run species ranging from helium to uranium. A future upgrade may allow polarized ${ }^{3} \mathrm{He}$ beams as well. Beam current ranges from $10 \mathrm{uA}$ at EBIS injection to $15 \mathrm{~mA}$ at the EBIS source extraction.

With the improvements of species switching time and the wide range of species that will be available with the new EBIS, the RHIC and NSRL programs will be able to operate in an increasingly efficient manner by reducing delays in switching time and tuning. Both programs will also be able to take advantage of the capability of EBIS to produce ions that the Tandem cannot currently generate.

\section{DIAGNOSTICS}

Figures 1 and 2 depict the locations of the diagnostics that will be used in the EBIS pre-injector. A standard set of diagnostics will be installed and include faraday cups (FC), a fast faraday cup (FFC), current transformers (CT), profile monitors (PM), and an emittance pepperpot (PP).

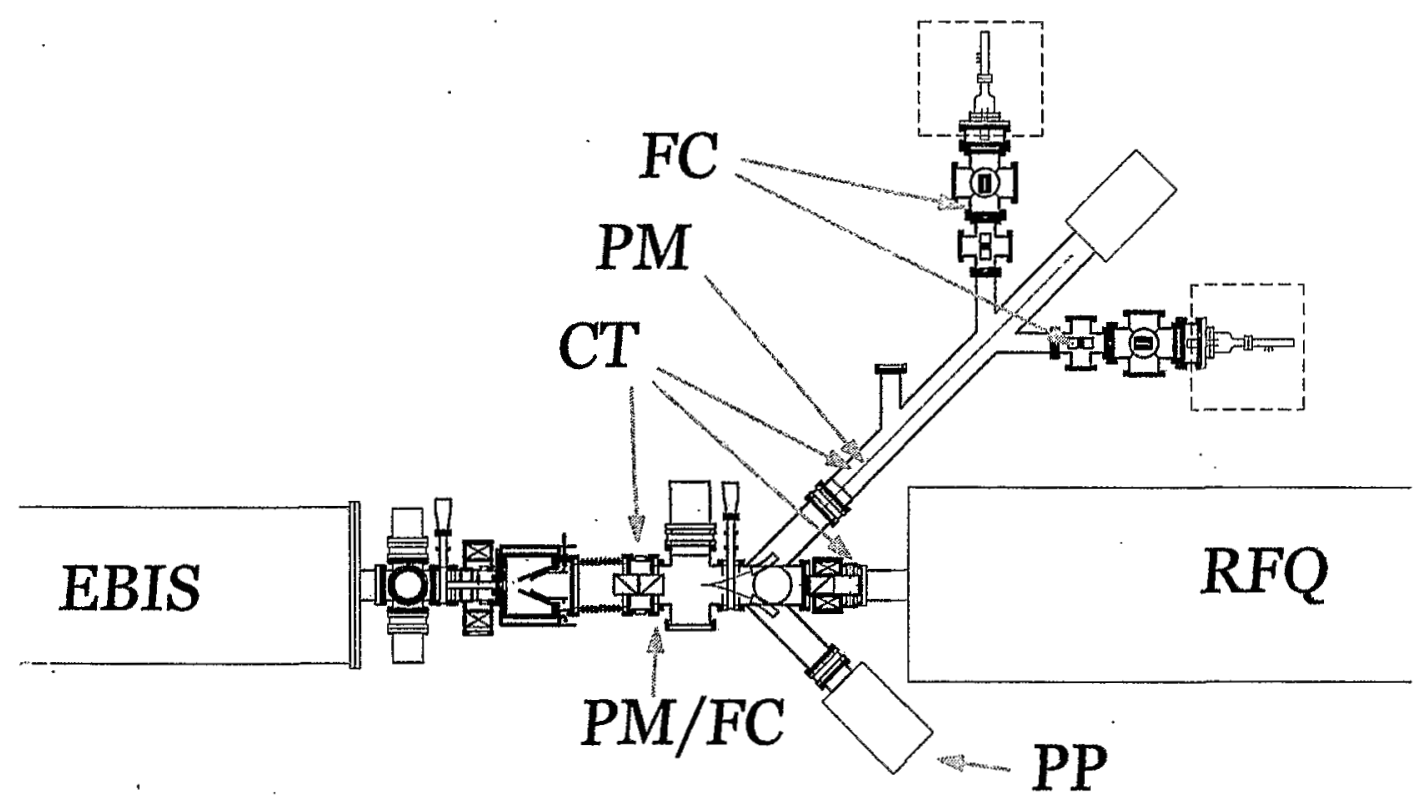

FIGURE 1. Layout of diagnostics in the external ion injection lines (EIL) and in the low energy beam transport (LEBT) 


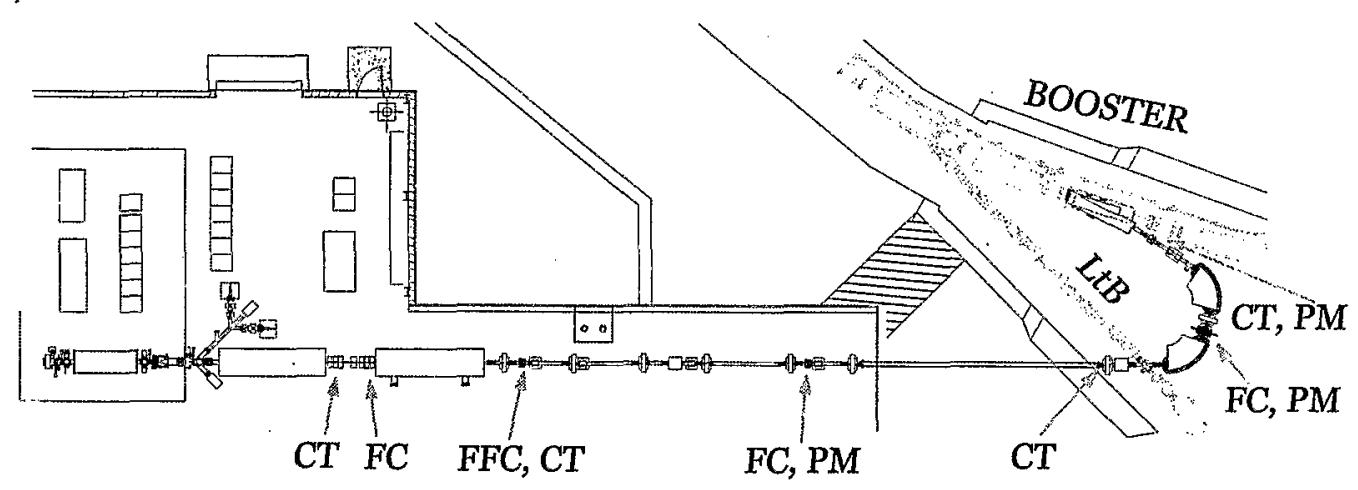

FIGURE 2. Layout of diagnostics in the transfer line to Booster

\section{Faraday Cups}

Six faraday cups and one fast faraday cup will be distributed among the pre-injector and transfer line.

The faraday cups are pneumatically inserted into the beamline aperture to make a measurement. The accumulated electric charge is detected as a current and then processed by front end electronics. It is desired for the current measurement to have a resolution of $0.1 \mathrm{uA}$. The resulting current measurement and pulse waveform will be digitized and input into the Controls system for distribution, viewing, and logging. An oscilloscope will be used for digitizing the fast analog pulse waveforms and an MADC will be used to digitize the current measurement. Since the insertion of the faraday cup is a destructive measurement device, it can also be used as a beam stop.

The fast faraday cup installation will make use of an existing unit that was removed from another beamline. This unit has bandwidth in the $\mathrm{GHz}$ range that will allow the $100 \mathrm{MHz}$ bunch structure of the beam to be observed. A scope will be used to digitize this signal.

\section{Current Transformers}

Seven current transformers will be installed as shown previously in Figures 1 and 2. The transformers will be fabricated at BNL and be similar to the transformers currently installed in the Tandem to Booster (TTB) and test EBIS lines. Six of the transformers will be identical in size with the seventh transformer at the entrance to the EBIS trap being slightly larger to accommodate the beamline components. At present, two transformers in the EIL and the one transformer at the exit of the RFQ will be mechanically mounted inside conflat flanges which will help in keeping the space requirements low. The remainder of the transformers in the transport line will have specially designed enclosures.

A one turn calibration winding will be installed around each transformer to assist in measuring each transformer's response independent of beam conditions. Electronics similar to those used for the TTB transformers will be used. The signals will be 
digitized using an oscilloscope and input into the Controls system for remote viewing. Data will be taken on a pulse by pulse basis, if possible. Otherwise, light averaging will be done to obtain the measurements with resolution of $0.1 \mathrm{uA}$.

Figure 3 depicts the progressive fabrication of the transformers beginning at the bottom right with the bare high permeability ferrite core. Moving towards the left in the bottom row, the picture shows the core being wound with 28 gauge insulated wire. Moving on to the top right, the core is shown with kapton tape and continuing left displaying the TTB style mechanical enclosures.
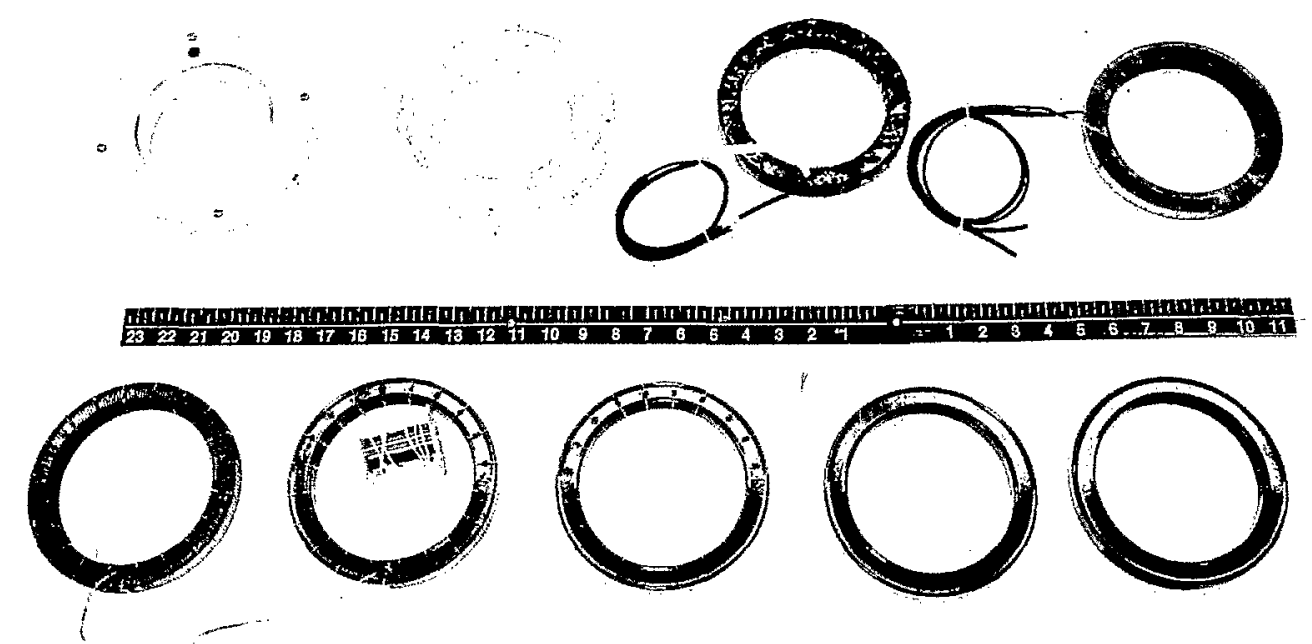

FIGURE 3. Fabrication of current transformers

\section{Profile Monitors}

Beam profile measurements will be made at four locations and be installed in conjunction with the faraday cups on dual feedthroughs at two locations. A fifth profile monitor is already installed at a location in the existing TTB line, just past the transport line bending magnets. Two $32 \mathrm{H} \mathrm{x} 32 \mathrm{~V}$ multiwire assemblies will be moved from the TTB line and installed in EBIS. The required remaining multiwire assemblies will be purchased. The $0.1 \mathrm{~mm}$ thick wires in the units are made of Tungsten-Rhenium (W-Re). Units will be pneumatically inserted into the beamline when profile measurements are needed.

The profile measurements are obtained by processing the current signal that is generated when the beam interacts with the wires, with a resolution of $1 \mathrm{~mm}$. These currents are processed by integrators and then input into an MADC for digitization and display of the results.

\section{Emittance Pepperpot}

A pepperpot device that can measure the emittance of an ion beam in a single pulse is being developed on the EBIS test stand for eventual use in the EBIS pre-injector ${ }^{4}$. The pepperpot schematic is shown in Figure 4. A mask with an array of small holes is inserted into the beam. An image of the beam passing through the small holes is 
holes is generated on a phosphor screen behind the mask which is then digitized and captured by a CCD camera. In addition, a microchannel plate can be placed between the mask and phosphor screen to enhance the image intensity and to gate the measurement such that measurements can be made at a specific time by pulsing the voltage on the microchannel plate. The two dimensional profiles captured by the camera are then used to calculate a four dimensional beam emittance by use of software.

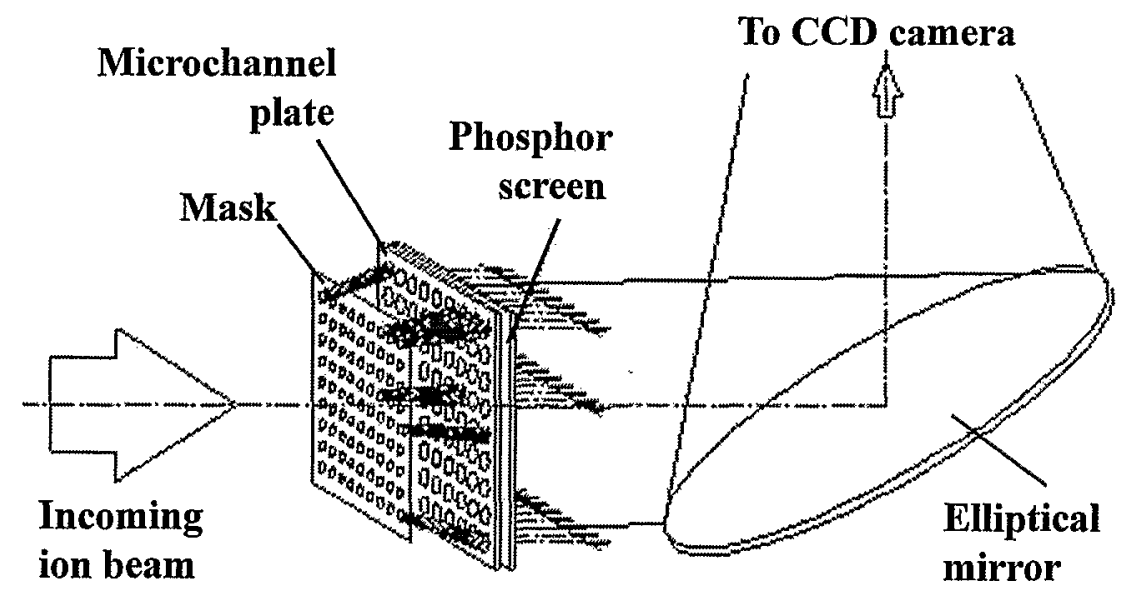

FIGURE 4. Schematic of the Pepperpot device

With a nominal beam diameter of $50 \mathrm{~mm}$, it was determined that the array of holes will be $25 \times 25$ with a distance between the individual holes around $2 \mathrm{~mm}$. By using a CCD camera with resolution of $1000 \times 1000$, each small beamlet that travels through the mask hole will cover 40 channels on the camera. Calibration can be performed by shining a laser beam through the mask. Initial results from the pepperpot installed on the EBIS test stand have been very good. Figure 5 shows a calculated horizontal emittance of Xe-132. Resolution of the measurements is expected to be around 0.5 mrad in divergence and $0.1 \mathrm{~mm}$ in size.

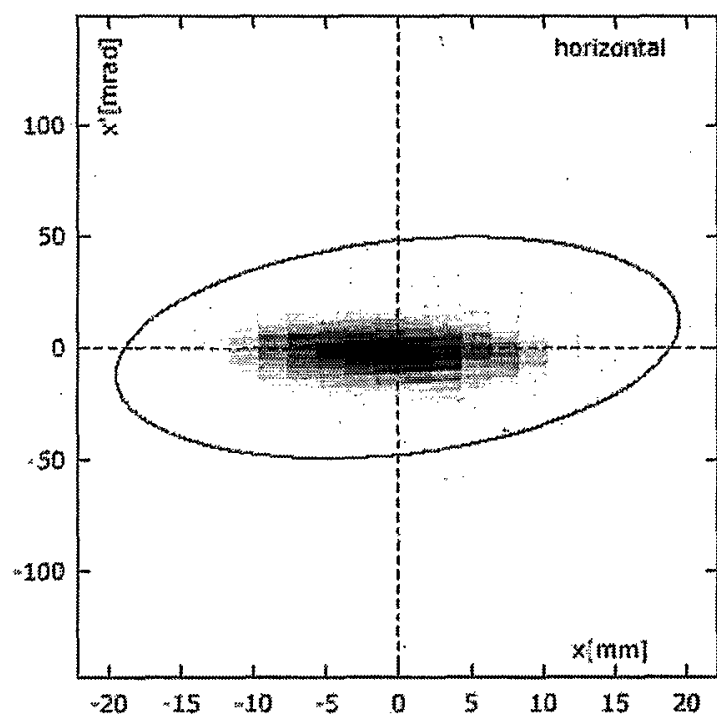

Isotope: $X^{2}-132$

PMS emittance: $232.711 \mathrm{~mm} \cdot \mathrm{mrad}$ Norm. RMS emittance: 0.13619 mm.mrad Aplat: 0.2589 Beta: $0.4068 \mathrm{~mm} / \mathrm{nurad}$ Gamna: $2.623 \mathrm{mrad} / \mathrm{mm}$

s:-centrold: $0.577 \mathrm{~mm}$ xasigma: $9.730 \mathrm{~mm}$

$x^{2}$-centroid: $\$ 99.320$ mrad $x^{*} \sim$ sigma: $24.709 \mathrm{mizd}$

FIGURE 5. Results from the EBIS Test Stand Pepperpot 


\section{CONTROLS INTERFACE}

In general, the EBIS Controls system will be an extension of the existing RHIC Controls system. The Diagnostics-Controls interface is no exception and will make use of the VME-based standard RHIC system. Hardware control will be provided by digital output boards, trigger boards, and timing distribution boards. Status information and signal monitoring will be done by digital input boards, MADCs, and an oscilloscope. Multiplexers will be used for the scope input channels as all signals will not need to be viewed at the same time. For example, if a faraday cup is inserted into the beamline aperture, devices downstream of that cup will not measure any useful signal. A software user interface will be provided through the BNL-developed pet program. Standard software programs for displaying and logging data already exist for most of the diagnostics with the exception of the pepperpot. These existing programs will simply need the addition of the new devices into the programs' databases for proper operation. The pepperpot has a standalone software program which could eventually be integrated into the Controls system.

\section{CONCLUSION}

The new EBIS pre-injector will provide many additional benefits to the RHIC and NSRL programs. As with any pre-injector line, a set of diagnostics will be crucial to determining proper operation of the EBIS. The proposed suite of diagnostics will be similar to diagnostics used elsewhere in the facility wherever possible to maximize value.

\section{REFERENCES}

1. J. G. Alessi, et al, "Design of an EBIS for RHIC", in Proceedings of the Particle Accelerator Conference 2003, (IEEE, Portland, Oregon, 2003).

2. J. G. Alessi, et al, "Progress on Test EBIS and the Design of an EBIS-Based RHIC Preinjector", in Proceedings of the Particle Accelerator Conference 2005, (IEEE, Knoxville, Tennessee, 2005).

3. J. Alessi, et al, "Electron Beam Ion Source Pre-Injector Project (EBIS) Conceptual Design Report", September 2005.

4. E. Beebe, J.G. Alessi, A. Pikin, L. Liljeby, "Pepperpot Emittance Measurement Device", Technical Note, 2005. 